\title{
Increasing interactivity in IPTV using MPEG-21 descriptors
}

\author{
Christos-Nikolaos Anagnostopoulos ${ }^{1}$, George Tsekouras ${ }^{\mathrm{I}}$, Damianos \\ Gavalas $^{l}$, Daphne Economou ${ }^{1}$ and Ioannis Psoroulas ${ }^{2}$ \\ 1 University of the Aegean, Cultural Technology and Communication \\ Dpt., 5 Sapfous Str., Mytilene, Greece, 81100 , \\ \{canag,gtsek,dgavalas.d.economou\}@ct.aegean.gr \\ 2 National Technical University of Athens, Electrical and Computer \\ Engineering Dpt., NTUA Campus, Athens, Greece \\ psoroulas@telecom.ntua.gr
}

\begin{abstract}
This paper introduces a novel approach for authoring a diversity of multimedia resources (audio, video, text, images, etc). An authoring tool (Developer21) supporting a metadata model according MPEG-21 XML schema files is described. The main scope of Developer2 1 is to increase interactivity resulting from the use of metadata in several broadcast scenarios in interactive or IP television. In addition to basic functionalities (editing and enrichment of audiovisual contents), innovative content management functionalities of the Developer 21 are briefly presented. As the focus of this paper was to provide a brief description of a novel authoring tool for metadata and descriptor management, only core functionalities are presented.
\end{abstract}

\section{Introduction}

The number of available digital contents is increasing over Internet and broadcast networks. Such a quantity of documents requires new ways to handle them. Besides, new services need tools to describe and organize these documents in an efficient and extensible way. These issues make metadata an interesting subject of research. Metadata are "information about data" and can include characteristics about the data such as the content, accuracy, reliability and the source. Metadata provide the mechanism to describe data in a consistent form which allows users to gain a uniform understanding of the content and fitness for purpose of datasets. Metadata have many applications and they can be used to:

\footnotetext{
Please use the following format when citing this chapter:

Anagnostopoulos, C.-N., I'sekouras, G., Gavalas, D., I:conomou, D., Psoroulas, I., 2007, in IFIP International Federation for Information Processing, Volume 247, Artificial Intelligence and Innovations 2007: From Theory to Applications, eds. Boukis, C., Pnevmatikakis, L., Polymenakos, L., (Boston: Springer), pp. 65-72.
} 
- concisely describe datasets and other resources using elements such as the name of the dataset, the quality, how to access the data, what is the purpose of data and other related information

- enable effective management of resources

- enable accurate searching and resource discovery

- provide an online interface to a dataset and link to other information about it

- accompany a dataset when it is transferred to another computer so that the dataset can be fully understood, and put to proper use.

The majority of metadata specifications use the eXtensible Markup Language (XML) [1]. The XML language permits to build hierarchical structures suitable for most types of documents and therefore facilitates advanced search. XML is often used with XML schemas [2]. An XML schema is an XML language expressing rules to create XML documents. Most metadata standards for multimedia content are built on this language, among them MPEG-7 [3], MPEG-21 [4]. The primary goal of metadata is to manage the huge number of digital sources, thus facilitating the search. However, metadata is employed for many other functions. Metadata is useful to give information about multimedia resources. For instance, information can be displayed on the client screen in interactive TV while playing the audiovisual content. This also can be applied in all applications of IPTV.

Therefore, metadata are quite useful for building interactive interfaces. Such devices collect multiple mixed multimedia contents inside the same structure. Metadata defines the spatial and temporal layout as well as hyperlinks. Examples of such interactive interfaces lie in DVD movies where images, texts, audio tracks, audiovisual sequences are combined. Another example of such interactivity could be when a character appears in a movie, a textual description of this character is displayed and a hyperlink is proposed to see a biography of this actor. Metadata is also used for content protection which restricts content usage to a particular user or group of users; it is particularly useful for service providers in a pay-per-view scenario. Finally, adaptation can be performed by using metadata; In this case metadata describes terminal capabilities, network characteristics and the way data has to be modified.

In this paper an authoring tool under the name Developer21, which supports the MPEG-21 standard is described. Section 2 shows similar works and gives the background of MPEG-21, which is the standard implemented in this tool. The main characteristics and the scopes of Developer 21 are described in Section 4. Finally, the last section concludes this paper.

\section{Literature-market review}

Surprisingly, very few authoring tool based on the MPEG-21 standard are present in the market as well as in the literature. In contrast, several metadata authoring tools based on the MPEG-7 standard have been exhaustively described and presented in the market [5-8]. 
Enikos [9] designed a MPEG-21 authoring tool, called DIEditor, allowing users to link multiple resources inside a MPEG-21 structure. In addition, in the framework of ENTHRONE project [10], the M-Tool was created combining and unifying TVAnytime and the MPEG-21 standard. In the latter work, MPEG-21 provided content protection, network adaptation, client terminal adaptation and a structure to link several multimedia contents while TVAnytime provided content-descriptive metadata and temporal segmentation.

MPEG-21 [4] aims at defining an open framework for the delivery and consumption of multimedia contents in heterogeneous conditions. In other words, the goal of MPEG-21 is to define a metadata model to support users to exchange, access, consume and manipulate Digital Items in an efficient, transparent and interoperable way. MPEG-21 is based on two essentials concepts: the definition of a fundamental unit of distribution and transaction (called the Digital Item or DI) and the concept of users interacting with Digital Items.

The MPEG-21 specification is flexible and enables higher level functionality and interoperability by allowing the connection of the several parts of MPEG-21, the inclusion of other description schemes, etc. The DID [11] represents a complete separation of metadata from its associated media resource. The DID specifications encompass the following features:

- the Digital Item Declaration Model,

- the Digital Item Declaration Representation in XML and

- XML schemas comprising grammars for the Digital Item Declaration representation in XML

The Digital Item Identification (DII) schema (MPEG-21, part 3) [12] uniquely identifies Digital Items and parts thereof, relationship between Digital Items (and parts thereof) and relevant description schemes. The 4th part of MPEG-21 [13] defines an interoperable framework for Intellectual Property Management and Protection (IPMP). The framework includes standardized ways of retrieving IPMP tools from remote locations, exchanging messages between IPMP tools and between these tools and the terminal.

MPEG-21 Intellectual Property Management and Protection (IPMP) manages rights and intellectual property of a specific resource. It also addresses authentication of IPMP tools, and has provisions for integrating Rights Expressions according to the Rights Expression Language REL [14] and the Rights Data Dictionary (RDD) [15], which are MPEG-21 parts 5 and 6 respectively.

MPEG-21 Right Expression Language (REL) specifies whether a given group of people can perform a given right upon a given resource under a given condition. Finally, MPEG-21 Digital Item Adaptation (DIA) [16] has recently been finalized as part of the MPEG-21 Multimedia Framework. DIA specifies metadata for assisting the adaptation of Digital Items according to constraints on the storage, transmission and consumption, thereby enabling various types of quality of service management. 


\section{The MPEG-21 Authoring tool (Developer21)}

\subsection{Problem Description}

Developer21 for MPEG-21 serves as a multimedia authoring tool adding or extracting MPEG-21 descriptors and metadata in various multimedia assets as shown in Figure 1. Once created, these descriptors (in XML schema files) are locally stored and its list is displayed to the user. The user is then allowed to select one or more descriptor schema which can be further processed and visualized using the respective graphical user interface (GUI). Users have the possibility to create a new MPEG-21 Digital Items, edit, delete, convert or send this metadata document to a specific (local or external) metadata database.

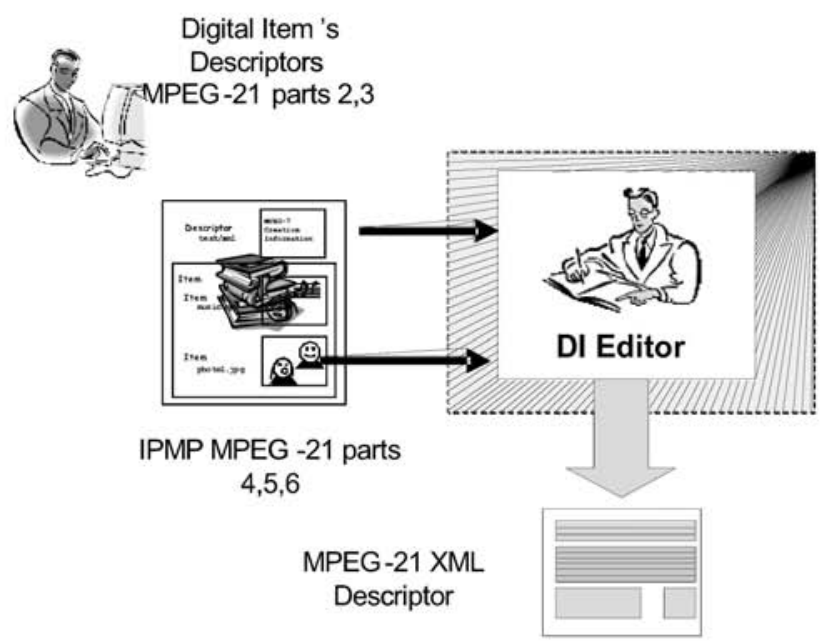

Fig. 1. The operation of Developer21 for authoring XML descriptors.

MPEG-21 enables the hierarchical representation of multimedia contents which is useful to create advanced and interactive multimedia contents. Every resource is described using the MPEG-21 standard and more specifically through the relevant XML schema which is provided by MPEG-21 standard. Developer21 is designed to support 6 different XML schemas, each one dedicated to the respective MPEG-21 part. The MPEG-21 descriptors that are provided by Developer21 are the following:

- Digital Item Declaration

- Digital Item Identification

- Intellectual Property Management and Protection

- Rights Expression Language

- Rights Data Dictionary

- Digital Item Adaptation 
DID was almost totally covered in Developer21 as it is the core definition part of the MPEG-21standard, including all the necessary architecture for the creation, the exchange and the manipulation of the DIs. However, special emphasis was given to the protection of the Intellectual Property Rights and therefore important parts of MPEG-21's IPMP, REL and RDD schemas are supported.

Developer 21 is a prototype that provides all the necessary operations to create or manage metadata or descriptors relevant to multimedia assets. The main scope is to provide the end-user and content provider with a reference tool that enable annotating, browsing, maintaining, monitoring and querying multimedia descriptors.

According to MPEG-21, a user is anyone that interacts with Digital Items. Hence, a User can be an individual, an organization, corporation, any community, consortium or even a government. Moreover, Users act in various roles including creators, consumers, rights holders, content providers, distributors, etc. Therefore, it is designed to help them in annotating and authoring multimedia resources. In other words, this application allows users to create objects' relationships to each other in order to enrich multimedia resources with metadata both formatted and stored in $\mathrm{XML}$ or in a relational database. This type of service is useful for applications that present a mixture of textual, graphical, and audio data.

\subsection{Modules of Developer21}

Developer2 1 is composed of two modules, namely the Digital Item editor (DI editor) and Digital Item Manager (DI manager). It is a Digital Item Declaration model editor, Digital Item generator and Digital Item Browser. A DI is edited or generated by inserting, deleting or modifying metadata on account of the DID, DII, IPMP, RDD, REL and DIA specifications.

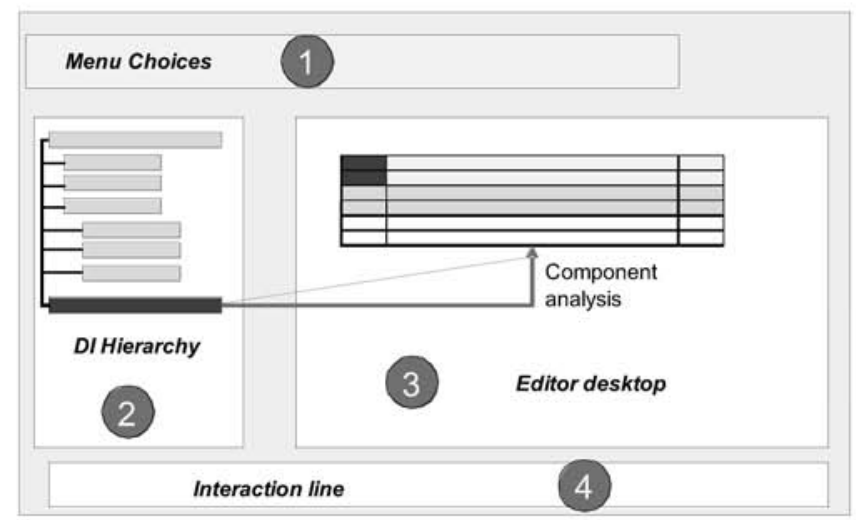

Fig. 2. The architecture of Developer21's editor panel.

When a user requests to create a new Digital Item, DI editor is invoked to create the MPEG-21 structure according the respective part of this standard. For example, if 
the user intends to create a very simple DI, only MPEG-21 part 2 and the relevant $\mathrm{XML}$ is sufficient. However, if Intellectual Property Rights need to be specified, the user should use the respective protection model which is defined in MPEG-21 part 4. In any case, the DID generation is achieved through a graphical representation of the MPEG-21 structure in explorer-like panel as shown in Figure 2. New elements are simply added by performing a drag and drop from the desktop to the MPEG-21 structure. These elements consist of MPEG-21 structure elements (Container, Item and Component) and Descriptor elements (REL, RDD and DIA metadata).

The MPEG-21 DI editor is composed of four areas as represented in Figure 2, the Menu choices (1), the relevant hierarchy and tree visualization (2), the editor desktop where the manipulation of elements takes place (3) and the user-tool interaction line where messages generated from the software appear (4). Similarly to the creation of a new MPEG-21 document, updates to the current document are achieved by dragging and dropping different icons from area 3 to area 2 . The tree view in area 2 gives the XML structuring components while area 3 gives the details of each selected component. Selecting fields in the top tree displays its full content in the bottom tree.

Basic information about descriptors is provided by the graphical representation: the type of descriptor (DID,DII, IPMP, RDD, REL, DIA), the type of program information (general information or only audio and video attributes). The schema file can be selected from a pull-down menu in the menu bar. Selecting a particular MPEG21 part triggers the appropriate XML schema for edition and visualization.

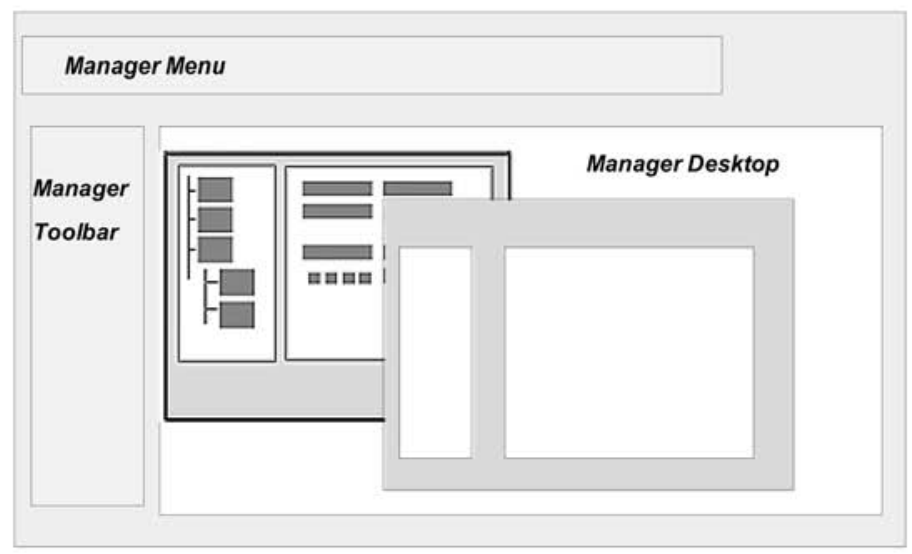

Fig. 3. The architecture of Developer21's manager panel.

\subsection{Increasing interactivity in IPTV with Developer21}

In parallel with the editing and browsing capabilities of theDeveloper 21 tool, metadata management is also supported. Binding of metadata and XML descriptors with the actual multimedia content is performed in order to create the integrated Digital Item that contains the actual content and the descriptive information. The 
manager is able for controlling, refreshing and synchronizing metadata files in conformance with their content including detection and elimination of XML metadata (sub-)item (or component) duplicates, and checking freshness and consistency of both metadata associated to distributed digital resources.

When a Digital Item is processed with DI Manager (see Figure 3), it is in the appropriate form to interact with an Expert System that is currently implemented for increasing the interactivity in IPTV or iTV. The expert system will be able to assign a TV viewer to a specific social category and then match the appropriate audiovisual content according the respective MPEG-21 descriptors. The whole architecture is depicted in Figure 4.

In general, personalization allows users to browse programs much more efficiently according to their preferences. On the other hand, personalization also enables to build social networks that can improve the performance of current IPTV systems considerably by increasing content availability, trust and the realization of proper incentives to exchange content.

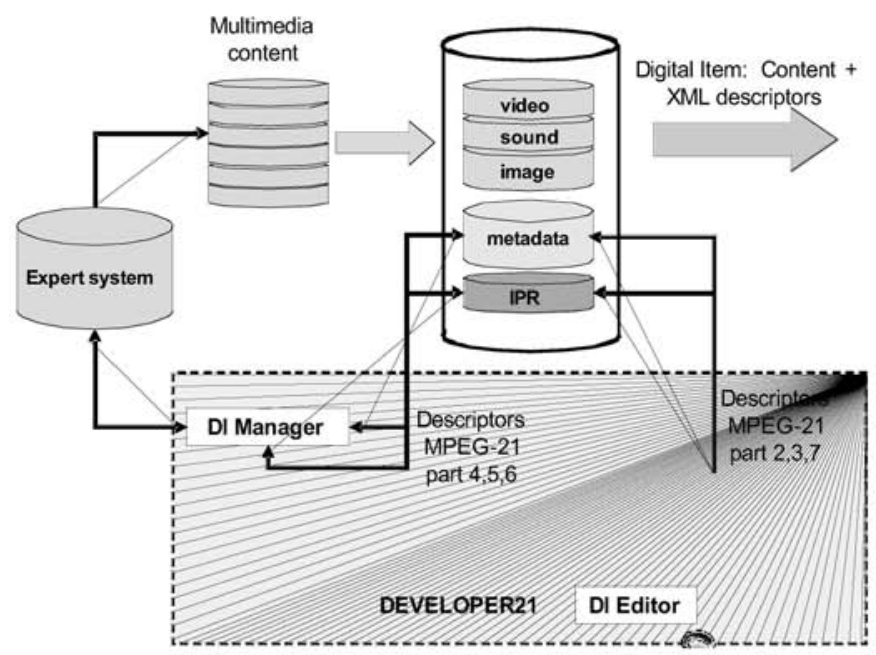

Fig. 4. The operation of Developer21 for authoring XML descriptors joined with an expert system for content personalization.

\section{Conclusions}

In this paper we have proposed an novel authoring tool implementing MPEG-21 XML schemas for modeling and managing content descriptive metadata associated to audiovisual resources. The functionalities of the authoring tool have been presented and associated with content personalization and improvement of interactivity in IPTV. The novelties introduced by this work is also highlighted as to 
our knowledge Developer 21 is the only MPEG-21 authoring tool capable to produce and manage DI that support IPMP, REL and RDD schemas.

Acknowledgements: This work is supported by the General Secretariat of Research and Technology (Project "Software Application in Interactive Kids TVMPEG-21", project framework "Image, Sound, and Language Processing", project number : ЕНГ-16). The participants are the University of the Aegean, the Hellenic Public Radio and Television (ERT) and the Time Lapse Picture Hellas.

\section{References}

1. eXtensible Markup Language (XML), http://www.w3.org/XML

2. XML Schema, http://www.w3.org/XML/Schema

3. ISO MPEG-7, part 8: Information technology -- Multimedia content description interface -- Part 8: Extraction and use of MPEG-7 descriptions, ISO/IEC TR 15938-8:2002.

4. ISO MPEG-21, part1: Information technology - Multimedia framework (MPEG-21) Vision, Technologies and Strategy, ISO/IEC TR 21000-1:2004.

5. J.M. Martinez, "Standards - MPEG-7 overview of MPEG-7 description tools", part 2, IEEE Multimedia, Volume 9, Issue 3, July-Sept. 2002 Page(s):83 - 93

6. Jae-Ho Lee, Gwang-Gook Lee, Whoi-Yul Kim, "Automatic video summarizing tool using MPEG-7 descriptors for personal video recorder" IEEE Transactions on Consumer Electronics, Volume 49, Issue 3, Aug. 2003 Page(s): 742 - 749

7. D. Bulgarelli, R. Cucchiara, C. Grana, R. Vezzani, "A Semi-Automatic Video Annotation tool with MPEG-7 Content Collections", Multimedia, 2006. ISM'06. Eighth IEEE International Symposium on Dec. 2006 Page(s):742 - 745

8. J.A. Lay, Ling Guan, "SOLO: an MPEG-7 optimum search tool", IEEE International Conference on Multimedia and Expo, vol. 2, 30 July-2 Aug. 2000 pp. $777-780$.

9. Enikos DI Creator, http://www.enikos.com. Last accessed on the 1th of April 2007.

10. Boris Rousseau, Wilfried Jouve, Laure Berti-Équille, "Enriching Multimedia Content Description for Broadcast Environments: From a Unified Metadata Model to a New Generation of Authoring Tool", Proc. of the 7th IEEE International Symposium on Multimedia (ISM'05), 2005, pp.8-15.

11. ISO MPEG-21, part 2: Information technology - Multimedia framework (MPEG-21) Digital Item Declaration, ISO/IEC TR 21000-2, 2nd Edition, 2005.

12. ISO MPEG-21, part 3: Information technology - Multimedia framework (MPEG-21) Digital Item Identification, ISO/IEC TR 21000-3, 1st, 2003.

13. ISO MPEG-21, part 4: Information technology - Multimedia framework (MPEG-21) Intellectual Property Management and Protection Components, ISO/IEC TR 21000-4, 1st Edition, 2006.

14. ISO MPEG-21, part 5: Information technology - Multimedia framework (MPEG-21) Part 5: Rights Expression Language, ISO/IEC TR 21000-5, 1st Edition, 2004.

15. ISO MPEG-21, part 6: Information technology - Multimedia framework (MPEG-21) Part 6: Rights Data Dictionary, ISO/IEC TR 21000-6, 1st Edition, 2004.

16. ISO MPEG-21, part 7: Information technology - Multimedia framework (MPEG-21) Part 7: Digital Item Adaptation, ISO/IEC TR 21000-7, 1st Edition, 2004. 